\title{
A COMPARATIVE STUDY ON THE USER EXPERIENCE OF PC GAMING VS CLOUD GAMING
}

\author{
John Millton J \\ Student, \\ Master of Computer Applications - \\ Information Security Management Services, \\ Jain University - SCMS, \\ Bangalore, Karnataka, \\ India
}

Article DOI: https://doi.org/10.36713/epra4284

\begin{abstract}
Cloud gaming is gaining in popularity, but it's still unknown whether the user-experience it provides is sweet enough to enable cloud gaming to exchange with a traditional PC gaming and what are the key expectations of a gamer. The Major goal of our research presented during this paper is to collect objective, experimental data on this subject. We've executed a user study that exposed ourselves to at least one simple and one complex game using cloud gaming and PC gaming approaches. After a timed gaming session on both platforms we analyzed the impact of the sessions and compared our experiences. The results of our study show that for a run of the mill casual gamer there's not much difference between cloud gaming and PC gaming. Whereas, more matured gamers tend to favor PC gaming, especially on high-end PCs. Although, both user groups did select cloud gaming as a far better solution for a low-end hardware. The results of our experiments lead to a conclusion that cloud gaming, in its current form, isn't ready to replace the PC gaming, but it fills a distinct segment that PC gaming has no possibility of reaching and basically allowing people to play their favorite titles without having to take a position in high-end gaming hardware.
\end{abstract}

KEYWORDS: User Experience, Internet, Cloud-based Applications, Computer Games, Virtualized Applications.

\section{INTRODUCTION}

As Computer games have an extended lasting and developed presence within the entertainment market ever since the computer game crash of 1983. consistent with the PC Gaming Alliance, whose members include representatives from companies like AMD, Razer, Intel and more, the PC game sales have reached $\$ 18.6$ billion in 2011 , an $11 \%$ increase from 2010 , and they are said to rise to $\$ 25.5$ billion by 2015 [1]. To put it into perspective, the annual global movie ticket sales in 2011 have reached $\$ 32.6$ billion in 2011, which was only a $3 \%$ improvement from the year before 0 . The PC game market is closely tied to a different entertainment market which is that the computer game market, which was its predecessor with beginnings during a device called beam tube amusement device as early as 1947[3]. Personal computers, which are machines of varying specifications, intended for but not limited to gaming, gained the power to run basic games in early $1980 \mathrm{~s}$ with the discharge of computers like ZX Spectrum, the Atari 8-bit family and most notably Commodore 64 , which is that the best-selling computer in history. By that point computer game consoles - machines of fixed specifications intended exclusively for entertainment purposes, especially for enjoying games, were in their second generation. From the instant that PCs gained the gaming ability these two branches progressed on their own parallel timelines. Today computer game consoles are in their 7 th generation, represented by devices like the stationary PlayStation 4 or Xbox One and Mobile consoles like the Nintendo Switch. Personal computers, as they don't have fixed specifications, evolve on a more linear and natural scale, with constant improvement of processing power of CPUs and GPUs.

Since the birth of the cloud gaming idea in 2000 there are many platforms offering the cloud gaming service using various methods [7]. Several of them provide access to modern PC or console game titles, 
some to old games, some to other exclusive titles. They also vary on devices an individual can use to run them - PCs, consoles, TVs, smartphones. Most of those cloud gaming platforms serve only local markets, i.e. France or Taiwan. a couple of platforms that provide a worldwide service are:

- Gaikai - released in 2011, bought out in 2012 by Sony, it allowed playing modern and old PC and console games in a web browser. Is now available as the Sony PlayStation Now service.

- Google Stadia - released in 2020, Stadia is a cloud gaming service operated by Google. It is advertised to be capable of streaming video games up to $4 \mathrm{~K}$ resolution at 60 frames per second with support for highdynamic-range, to players via the company's numerous data-centers across the globe, provided they are using a sufficiently highspeed Internet connection.

- GeForce Now - Nvidia officially unveiling its name on September 30, 2015. GeForce Now lets users access a virtual computer, where they can install their existing PC games from existing digital distribution platforms and play them remotely.

- Project xCloud - xCloud currently runs via Microsoft's 54 Azure cloud computing centers, hosted in 140 countries. The service is designed to work with phones, either with touchscreen controls or Xbox controller over Bluetooth. It is currently in Early Access for a select user base. To be released in Q4 2020.

\section{PLATFORMS AND TECHNIQUES}

To compare PC gaming to cloud gaming we've used leading platforms of every type: Steam as the representative of the $\mathrm{PC}$ gaming and Project $\mathrm{xCloud}$ (Early Access) because the representative of the cloud gaming. Steam is currently the foremost advanced and therefore the hottest platform for digital distribution of games and offers a stimulating set of additional features. Other digital distribution platforms like Electronic Arts's Origin or Ubisoft's Uplay are trying to require the market share from it, but they still have a long way to go. Other companies like GOG.com use a special approach and offer a variety of games namely old games, which are hard to return by otherwise. Project xCloud cloud gaming service provides the simplest basis for comparison with access to modern PC game titles, as opposed to Stadia which has been reported to be faced with massive performance and Frame-rate issues.

\subsection{Objective gaming comparison}

The presented gaming comparison is predicated on quantitative technical measures and is supposed to point out whether there are any objective differences between PC gaming (playing a game downloaded from Steam) and cloud gaming (playing a game streamed through Project xCloud). The analysis is concentrated on the gaming experience only, leaving out the various additional features each platform offers. The games used for the test are "Assassin's Creed Odyssey" and "Cities:Skylines". These games were chosen due to significant differences in hardware requirements and their availability on both platforms. "Assassin's Creed Odyssey" is a demanding game, while "Cities:Skylines" is a game which should run smoothly even on a low-end PC.

Each game was played 2 times in 2 different settings:

1. High-end computer using Steam.

2. High-end computer using Project xCloud.

Due to the subjective nature of such a comparison, a group of quantitative attributes also as an inventory of expectations was prepared.

Each scenario was evaluated using the following list of questions:

1. How much data must be downloaded for the game to start? (Excluding the service client applications)

2. What is the average frame rate?

3. How much data does the game transfer in 5 minutes of gameplay?

4. Is it possible to change resolution?

5. Is it possible to change any graphics settings beyond resolution?

In order to measure the frame rates, the "FRAPS" video capture software was used. Internet traffic measurements were done by the "AIDA32" program. All measurements were performed on a $150 \mathrm{Mbit} / \mathrm{s}$ Internet connection (download/upload bandwidth), which ensured good performance of the xCloud service.

\subsection{User-experience comparison}

In contrast to the target game play comparison, the user-study was designed to live subjective impressions of players playing an equivalent game on a PC gaming platform (Steam) and a cloud gaming platform (xCloud). it was carried out on a group of 4 students all of which were presented the essential concepts of both systems and therefore the goal of the survey. They were asked to analyze the potential differences between the platforms, including visual differences, reaction time and their overall experience.

1. Please rate on a 1-10 scale how important are the aesthetics of the game to you.

2. Please rate on a 1-10 scale the fluidity of the game played in the traditional way.

3. Please rate on a 1-10 scale the fluidity of the game played using xCloud.

4. Please rate on a 1-10 scale the aesthetics of the game played in the traditional way. 
5. Please rate on a $1-10$ scale the aesthetics of the game played using xCloud.

6. Please rate on a 1-10 scale the response time of mouse and keyboard of the game played in the traditional way.

7. Please rate on a 1-10 scale the response time of mouse and keyboard of the game played using xCloud.

8. When playing the traditionally played game, did the screen ever refresh partially?

9. When playing the traditionally played game, did the screen seem blurry?

10. When playing the traditionally played game, did any artifacts show up (unexpected objects or stains)?

11. When playing the traditionally played game, was there any other quality loss?

12. When playing the game on $x$ Cloud, did the screen ever refresh partially?

\section{RESULTS AND COMPARISON}

\subsection{Objective gaming comparison}

The first area of interest that the experiment was Internet bandwidth usage. Two statistics are utilized in order analyze the differences - the quantity of downloaded data, which is required to run the game, and therefore the amount bandwidth consumed in 5 minutes of an average play. The results are presented in Table 1 and Table 2. Steam downloads a much bigger amount of data upfront and none little during the gameplay, while xCloud does the precise opposite - it requires no data downloaded upfront but requires a gentle stream of it during the gameplay. With a median rate of $500 \mathrm{MB}$ downloaded per 5 minutes, it might take about 1.5 Hours to surpass the $60 \mathrm{~GB}$ required to download the Steam version of "AC: Odyssey". whereas in the case of "Cities Skyline" (202.4 MB per 5 minutes with xCloud vs. 4 GB download with Steam) the edge would are reached within about 8.5 minutes. Comparing the 2 download rates of xCloud one can see that they're similar and mostly independent of the game, making the Steam game size the sole differentiating factor. This results in a conclusion that when bandwidth limitations are considered xCloud are going to be the higher choice for shorter game sessions which might require big amounts of knowledge to download otherwise, while for games that players plan to spend longer on, Steam would be the clear winner.

Table 1. How much data is required to be downloaded for the game to start? (Excluding the service client applications)

\begin{tabular}{|c|c|c|}
\cline { 2 - 3 } \multicolumn{1}{c|}{} & Cities Skyline & AC Odyssey \\
\hline Steam & $4 \mathrm{~GB}$ & $60 \mathrm{~GB}$ \\
\hline xCloud & $0 \mathrm{MB}$ & $0 \mathrm{MB}$ \\
\hline
\end{tabular}

Table 2. What is the average frame rate?

\begin{tabular}{|l|c|c|c|c|}
\cline { 2 - 5 } \multicolumn{1}{c|}{} & \multicolumn{2}{c|}{$\begin{array}{c}\text { Cities } \\
\text { Skyline }\end{array}$} & \multicolumn{2}{c|}{ AC Odyssey } \\
\cline { 2 - 5 } \multicolumn{1}{c|}{ Low } & High & Low & High \\
\hline Steam & 60 & 60 & 60 & 60 \\
\hline xCloud & 55 & 60 & 55 & 60 \\
\hline
\end{tabular}

Table 3. How much data does the game transfer in $\mathbf{5}$ minutes of gameplay?

\begin{tabular}{|c|c|c|}
\cline { 2 - 3 } \multicolumn{1}{c|}{} & Cities Skyline & AC Odyssey \\
\hline Steam & $0.156 \mathrm{MB}$ & $0.232 \mathrm{MB}$ \\
\hline xCloud & $202.4 \mathrm{MB}$ & $500.2 \mathrm{MB}$ \\
\hline
\end{tabular}


EPRA International Journal of Multidisciplinary Research (IJMR) - Peer Reviewed Journal

Volume: 6 | Issue: 4 | April 2020 || Journal DOI: 10.36713/epra2013 || SJIF Impact Factor: 5.614||ISI Value: 1.188

Table 4. Is it possible to change resolution?

\begin{tabular}{|l|l|l|}
\cline { 2 - 3 } \multicolumn{1}{c|}{} & Cities Skyline & AC Odyssey \\
\hline Steam & Yes & Yes \\
\hline xCloud & Yes & Yes \\
\hline
\end{tabular}

Table 5. Is it possible to change any graphics settings beyond resolution?

\begin{tabular}{|l|l|l|}
\cline { 2 - 3 } \multicolumn{1}{c|}{} & Cities Skyline & AC Odyssey \\
\hline Steam & Yes & Yes \\
\hline xCloud & No & No \\
\hline
\end{tabular}

\subsection{User-experience comparison}

Table 6 shows the user-experience comparison and the general results of the user survey collected after each user gaming session.

\begin{tabular}{|l|c|}
\hline \multicolumn{1}{|c|}{ Feature Tested } & Result Value \\
\hline F1: Average traditional gaming aesthetics (1-10) & 10 \\
\hline F2: Average XCloud aesthetics (1-10) & 7 \\
\hline $\begin{array}{l}\text { F3: Average traditional gaming mouse and keyboard response } \\
\text { times (1-10) }\end{array}$ & 10 \\
\hline F4: Average XCloud mouse and keyboard response times (1-10) & 8 \\
\hline F5: Traditional gaming partial screen refresh rate & $0 \%$ \\
\hline F6: XCloud partial screen refresh rate & $20 \%$ \\
\hline F7: Traditional gaming blurry screen rate & $0 \%$ \\
\hline F8: XCloud blurry screen rate & $10 \%$ \\
\hline F9: Traditional gaming artifacts occurrence rate & $0 \%$ \\
\hline F10: XCloud artifacts occurrence rate & $10 \%$ \\
\hline F11: Traditional gaming other quality loss rate & $0 \%$ \\
\hline F12: XCloud other quality loss rate & $5 \%$ \\
\hline
\end{tabular}

\section{CONCLUSION}

Thus, Results of our experiments also are not enough to easily select a far better platform. However, they hint at particular scenarios during which one among the choices performs better than the opposite. The data confirm the expected result that xCloud is best fitted to the low-end setups. The xCloud ability to beat hardware limitations and significantly improve the fluidity of the entire gaming experience made it the more preferred choice for low-end computers, no matter the standard loss. For high-end computers the winner can't be clearly selected. 
Basing on the gathered data it can be concluded that cloud gaming (represented by xCloud platform), in its current form, isn't ready to replace PC gaming (represented by Steam), but it definitely is in a position to reinforce gaming experience of casual gamers with low-end computers. Cloud gaming fills a distinct segment that PC gaming has no possibility of reaching - allowing people with budget computers to play their favorite titles without having to spend big amounts of cash on a specially tailored computer. The question remains whether that niche is large enough to form the cloud gaming concept survive actually and whether players are new to the scene, which it also caters to, wouldn't convert to traditional PC gaming at some point.

\section{REFERENCE}

1. A. Gallegos, "Year-over-year the PC continues to grow as a platform", ING UK, 2012. htttp://uk.ign.com/articles/2012/03/06/reportrecordpc-games-sales-in-2011

2. E. Orden, "Overseas Ticket Sales Buoyed Movies in 2011", The Wall Street Journal, 2012.

http://online.wsj.com/article/SB10001424052702 303812904577297784019921106.html

3. D.S. Cohen, "Cathode-Ray Tube Amusement Device - The First Electronic Game", http://classicgames.about.com/od/classicvideoga mes 101/p/CathodeDevice.html 